\title{
Testing Lorentz Invariance in QED (Part 2)
}

\section{Brett Altschul \\ University of South Carolina}

April 18, 2012

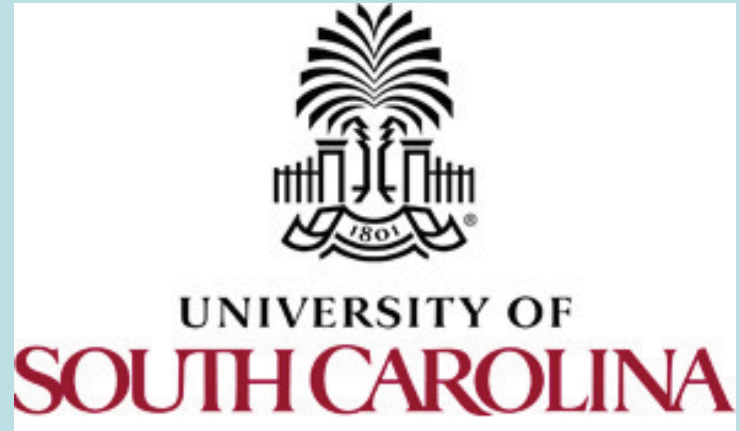


Atomic clocks are extremely important to tests of Lorentz symmetry, because atomic measurements can be made very, very precisely.

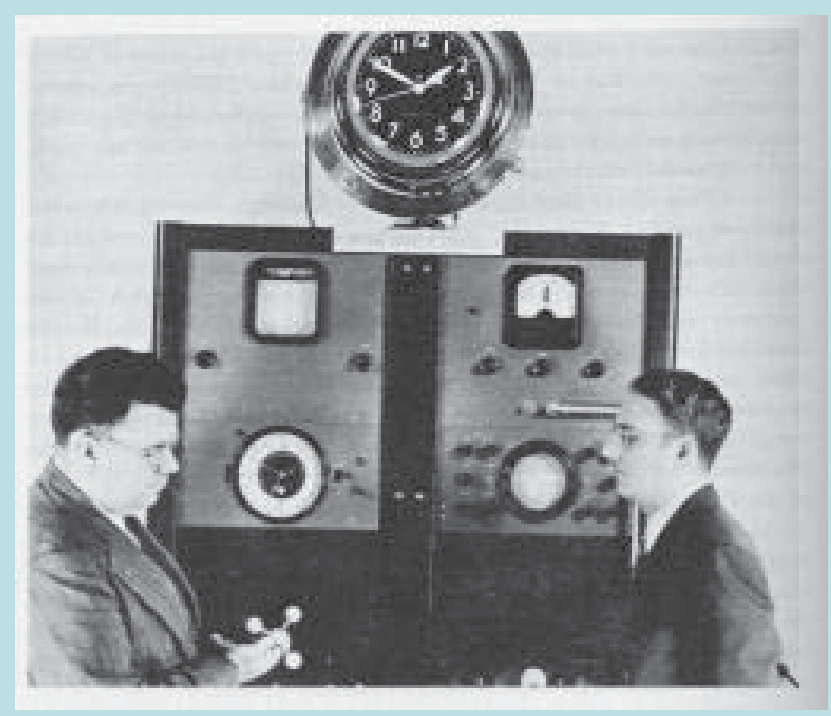

Edward Lyons built the first atomic clock with ammonia in 1949. 


\section{Outline}

- Introduction/Recap

- Atomic Clock Tests of Lorentz and CPT

- Ideas for Future Improvement

- Tests of Other Exotic Possibilities

- Conclusion

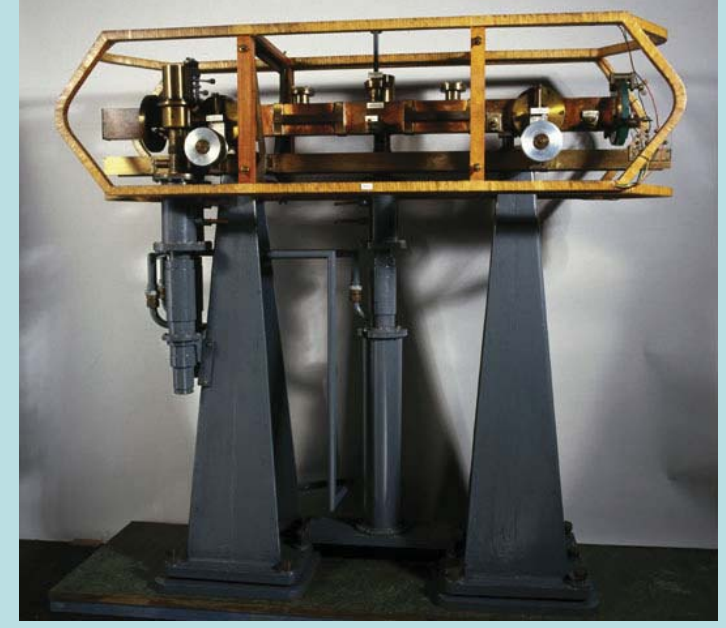

First Cs clock (1955) 


\section{Introduction}

There is presently a lot of interest in the question of whether Lorentz and CPT

symmetries are exact in Nature.

If violations of these fundamental symmetries are uncovered, they would be hugely important clues about the nature of new physics beyond the standard model.

The same holds for other forms of exotic physics, but Lorentz and CPT violation are the best understood possibilities. 


\section{Any (reasonable-looking) theory with CPT violation must also be Lorentz-violating.}

\section{[Greenberg, PRL 89, 231602 (2002)]}

So it would be good to have a systematic framework for studying any possible Lorentz and CPT violations. This framework is the standard model extension (SME), which uses the known tools of effective field theory to describe all possible forms of Lorentz violation involving standard model fields. 
Lorentz violating operators have objects built up from standard model fields, contracted with constant background tensors.

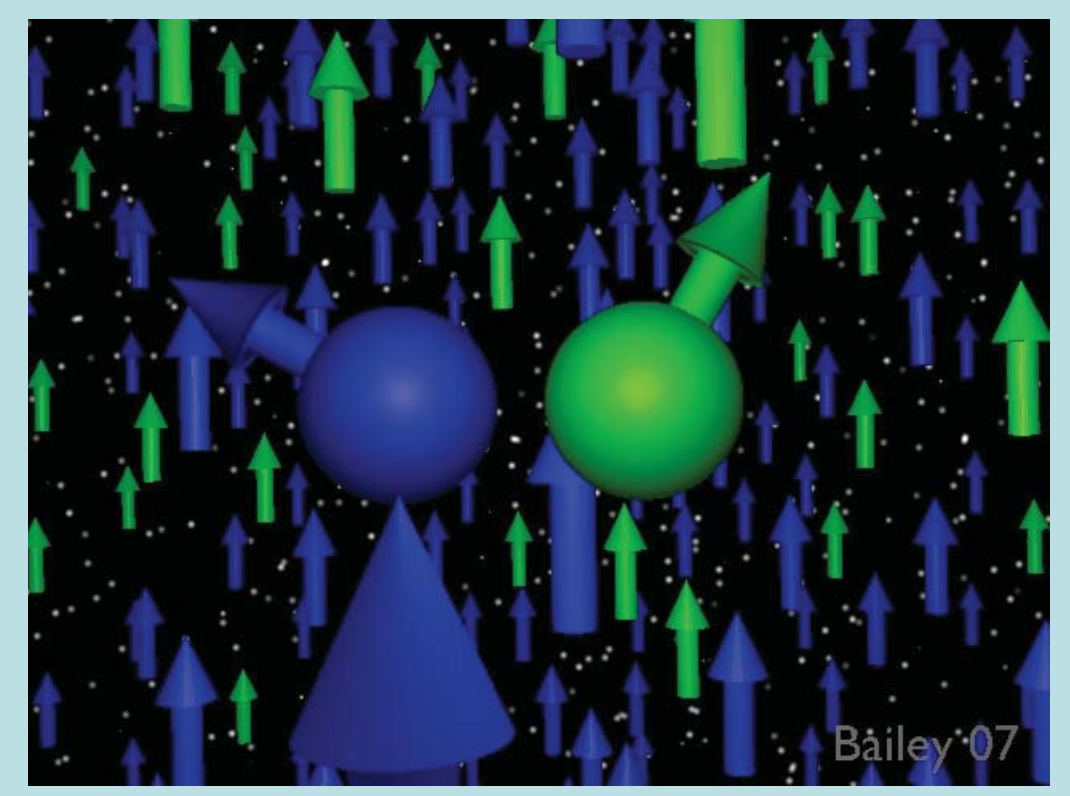

Earth-based laboratories will see slightly different local physics as the planet rotates and revolves. 
The Lagrange density for a Lorentz-violating free Fermion theory is:

$$
\begin{gathered}
\mathscr{L}=\bar{\psi}\left(i \Gamma^{\mu} \partial_{\mu}-M\right) \psi \\
M=m+a^{\mu} \gamma_{\mu}+b^{\mu} \gamma_{5} \gamma_{\mu}+\frac{1}{2} H^{\mu v} \sigma_{\mu v} \\
\Gamma^{\mu}=\gamma^{\mu}+c^{v \mu} \gamma_{v}+d^{v \mu} \gamma_{5} \gamma_{v}+e^{\mu}+i f^{\mu} \gamma_{5}+\frac{1}{2} g^{\lambda v \mu} \sigma_{\lambda v}
\end{gathered}
$$

$a, b, e, f$, and $g$ also violate CPT.

A separate set of coefficients will exist for every elementary particle in the theory. 
The photon sector contains more superficially renormalizable couplings.

$$
\mathscr{L}=-\frac{1}{4} F^{\mu v} F_{\mu \nu}-\frac{1}{4}\left(k_{F}\right)^{\mu \nu \rho \sigma} F_{\mu \nu} F_{\rho \sigma}+\frac{1}{2}\left(k_{A F}\right)^{\mu} \varepsilon_{\mu \nu \rho \sigma} A^{v} F^{\rho \sigma}
$$

Of the 23 independent coefficients, 14 of them give rise to photon birefringence in vacuum. These have been very tightly constrained by polarimetry measurements. (The polarization of light changes very little as it crosses the universe.) 
The remaining photon coefficients look like

$$
k_{F}^{\mu \nu \rho \sigma}=\frac{1}{2}\left(g^{\mu \rho} \tilde{k}^{v \sigma}-g^{\mu \sigma} \tilde{k}^{v \rho}-g^{v \rho} \tilde{k}^{\mu \sigma}+g^{v \sigma} \tilde{k}^{\mu \rho}\right)
$$

However, they can be defined away by a change of coordinates

$$
x^{\mu} \rightarrow x^{\prime \mu}=x^{\mu}-\frac{1}{2} \tilde{k}_{v}^{\mu} x^{v}
$$

This makes the photon sector conventional, but moves the LV to the matter sector. 
If we define away the non-birefringent photon $\mathrm{LV}$, the fermion coefficients become

$$
c^{\mu v} \rightarrow c^{\prime \mu v}=c^{\mu v}-\frac{1}{2} \tilde{k}^{\mu v}
$$

The physically observable quantity is $c^{\mu \nu}-\frac{1}{2} \widetilde{k}^{\mu v}$. This measures whether the natural coordinates in the electromagnetic sector are skewed relative to the natural coordinates for the fermions.

Other sectors have similar coefficients. 
The high-energy bounds I talked about last time are really bounds on these differences of coefficients in different sectors.

It is often convenient to simply define our coordinates so that the electromagnetic LV vanishes.

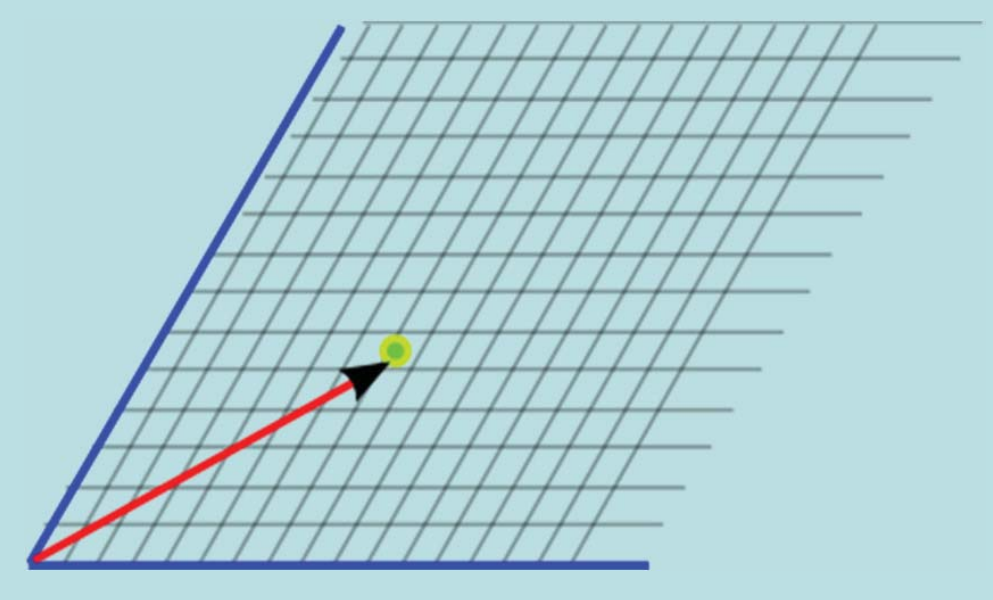


The same photon-matter differences can be measured in modern Michelson-Morley experiments.

These test whether the speed of light in a resonant cavity depends on the orientation of the cavity.
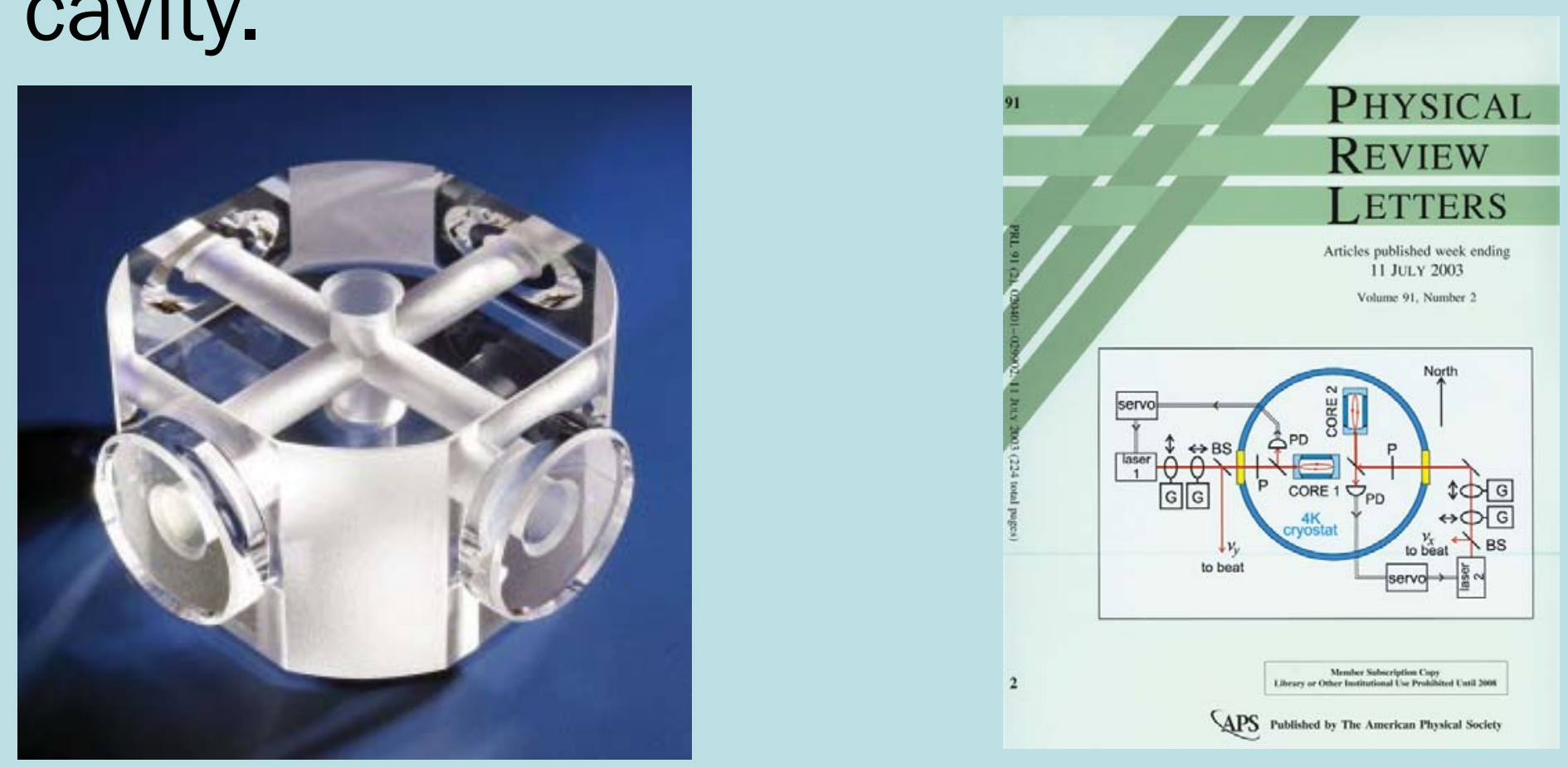
The current generation of Michelson-Morley tests use both optical and microwave, room temperature and cryogenic oscillator pairs.

These experiments can actually be used to test photon, electron, and nuclear Lorentz violation. The sensitivities to matter come from the fact that cavities may swell and contract along different axes. Different materials have different sensitivities to the matter-sector LV. 
The best Michelson-Morley bounds on (direction-dependent) changes in the two-way speed of light are at roughly the $10^{-17}$ level.

However, there aren't enough measurements using different materials to map out the whole parameter space, spanning multiple sectors.

There is thus significant room for experimental improvement using current state-of-the-art techniques. 


\section{Atomic Clock Tests}

Atomic clocks are good for Lorentz and CPT tests because of their high precision.

They are also useful because of their low energies. If the LV coefficients depend on energy (which they might), the high-energy astrophysical tests will be probing different parameters than low-energy experiments. 


\section{The nonrelativistic SME Hamiltonian can be determined by the usual method of Foldy- Wouthuysen transformations.}

$$
\begin{aligned}
H_{F W}= & \frac{p^{2}}{2 m}+\left[m\left(-c_{j k}-\frac{1}{2} c_{00} \delta_{j k}\right)\right] \frac{p_{j} p_{k}}{m^{2}} \\
& +\left(-b_{j}+m d_{j 0}-\frac{1}{2} m \epsilon_{j k l} g_{k l 0}+\frac{1}{2} \epsilon_{j k l} H_{k l}\right) \sigma^{j}+\left[a_{j}-m\left(c_{0 j}+c_{j 0}\right)-m e_{j}\right] \frac{p_{j}}{m} \\
& -\left[b_{0} \delta_{j k}-m\left(d_{k j}+d_{00} \delta_{j k}\right)-m \epsilon_{k l m}\left(\frac{1}{2} g_{m l j}+g_{m 00} \delta_{j l}\right)-\epsilon_{j k l} H_{l 0}\right] \frac{p_{j} \sigma^{k}}{m} \\
& +\left\{\left[m\left(d_{0 j}+d_{j 0}\right)-\frac{1}{2}\left(b_{j}+m d_{j 0}+\frac{1}{2} m \epsilon_{j m n} g_{m n 0}+\frac{1}{2} \epsilon_{j m n} H_{m n}\right)\right] \delta_{k l}\right. \\
& \left.+\frac{1}{2}\left(b_{l}+\frac{1}{2} m \epsilon_{l m n} g_{m n 0}\right) \delta_{j k}-m \epsilon_{j l m}\left(g_{m 0 k}+g_{m k 0}\right)\right\} \frac{p_{j} p_{k} \sigma^{l}}{m^{2}} .
\end{aligned}
$$


The Foldy-Wouthuysen transformations work much as usual, splitting $H$ into odd and even parts.

$$
H=m\left(\gamma^{0}+\mathcal{P}_{0}+\mathcal{O}_{0}+\mathcal{E}_{0}\right)
$$

Only the two parts of the Hamiltonian are much more complicated.

$$
\begin{aligned}
m \mathcal{P}_{0}:= & -p_{j} \gamma^{0} \gamma^{j} \\
m \mathcal{O}_{0}:= & {\left[-b_{0}+\left(d_{0 j}+d_{j 0}\right) p^{j}\right] \gamma_{5}+\left[a_{j}-\left(c_{j k}-c_{00} \eta_{j k}\right) p^{k}\right] \gamma^{0} \gamma^{j}+i f_{j} p^{j} \gamma_{5} \gamma^{0}+i\left[H_{0 j}+\left(g_{j 0 k}+g_{j k 0}\right) p^{k}\right] \gamma^{j} } \\
m \mathcal{E}_{0}:= & {\left[a_{0}-\left(c_{0 j}+c_{j 0}\right) p^{j}-m e_{0}\right]+\left[-b_{j}+\left(d_{j k}-d_{00} \eta_{j k}\right) p^{k}-\frac{1}{2} m \varepsilon^{k l m} \eta_{j m} g_{k l 0}\right] \gamma_{5} \gamma^{0} \gamma^{j} } \\
& -\left[m c_{00}+e_{j} p^{j}\right] \gamma^{0}-\left[\frac{1}{2} \varepsilon^{k l m} \eta_{j m} H_{k l}+m d_{j 0}-\varepsilon^{l m n} \eta_{j n}\left(\frac{1}{2} g_{l m k}-\eta_{k m} g_{l 00}\right) p^{k}\right] \gamma_{5} \gamma^{j}
\end{aligned}
$$


Only certain combinations of the SME coefficients are observable in the strictly nonrelativistic limit.

The SME coefficients multiply operators with derivatives and Dirac matrices. However, at low energies

$$
\begin{aligned}
& i \partial^{0} \approx m \\
& \gamma^{0} \approx 1
\end{aligned}
$$

The operators that differ by only these factors collapse into indistinguishability. 


\begin{tabular}{|c|c|c|}
\hline Symbol & Combination & Components \\
\hline$\tilde{b}_{J}$ & $b_{J}-\frac{1}{2} \varepsilon_{J K L} H_{K L}-m\left(d_{J T}-\frac{1}{2} \varepsilon_{J K L} g_{K L T}\right)$ & 3 \\
\hline$\tilde{b}_{J}^{*}$ & $b_{J}+\frac{1}{2} \varepsilon_{J K L} H_{K L}+m\left(d_{J T}+\frac{1}{2} \varepsilon_{J K L} g_{K L T}\right)$, & 3 \\
\hline$\tilde{b}_{T}$ & $b_{T}+m g_{X Y Z}$ & 1 \\
\hline$\tilde{g}_{T}$ & $b_{T}-m\left(g_{X Y Z}-g_{Y Z X}-g_{Z X Y}\right)$ & 1 \\
\hline$\tilde{H}_{X T}$ & $H_{X T}+m\left(d_{Z Y}-g_{X T T}-g_{X Y Y}\right)$ & 1 \\
\hline$\tilde{H}_{Y T}$ & $H_{Y T}+m\left(d_{X Z}-g_{Y T T}-g_{Y Z Z}\right)$ & 1 \\
\hline$\tilde{H}_{Z T}$ & $H_{Z T}+m\left(d_{Y X}-g_{Z T T}-g_{Z X X}\right)$ & 1 \\
\hline$\tilde{d}_{ \pm}$ & $m\left(d_{X X} \pm d_{Y Y}\right)$ & 2 \\
\hline$\tilde{d}_{Q}$ & $m\left(d_{X X}+d_{Y Y}-2 d_{Z Z}-g_{Y Z X}-g_{Z X Y}+2 g_{X Y Z}\right)$ & 1 \\
\hline$\tilde{d}_{J}$ & $m\left(d_{T, J}+\frac{1}{2} d_{J T}\right)-\frac{1}{4} \varepsilon_{J K L} H_{K L}$ & 3 \\
\hline$\tilde{d}_{Y Z}$ & $m\left(d_{Y Z}+d_{Z Y}-g_{X Y Y}+g_{X Z Z}\right)$ & 1 \\
\hline$\tilde{d}_{Z X}$ & $m\left(d_{Z X}+d_{X Z}-g_{Y Z Z}+g_{Y X X}\right)$ & 1 \\
\hline$\tilde{d}_{X Y}$ & $m\left(d_{X Y}+d_{Y X}-g_{Z X X}+g_{Z Y Y}\right)$ & 1 \\
\hline$\tilde{g}_{c}$ & $m\left(g_{X Y Z}-g_{Z X Y}\right)$ & 1 \\
\hline$\tilde{g}_{-}$ & $m\left(g_{X T X}-g_{Y T Y}\right)$ & 1 \\
\hline$\tilde{g}_{Q}$ & $m\left(g_{X T X}+g_{Y T Y}-2 g_{Z T Z}\right)$ & 1 \\
\hline$\tilde{g}_{T, J}$ & $m\left|\varepsilon_{J K L}\right| g_{K T L}$ & 3 \\
\hline$\tilde{g}_{D J}$ & $-b_{J}+m \varepsilon_{J K L}\left(g_{K T L}+\frac{1}{2} g_{K L T}\right)$ & 3 \\
\hline$\tilde{g}_{J K}$ & $m\left(g_{J T T}+g_{J K K}\right),($ no $K$ sum,$J \neq K)$ & 6 \\
\hline$\tilde{c}_{Q}$ & $m\left(c_{X X}+c_{Y Y}-2 c_{Z Z}\right)$ & 1 \\
\hline$\tilde{c}_{-}$ & $m\left(c_{X X}-c_{Y Y}\right)$ & 1 \\
\hline$\tilde{c}_{J}$ & $m\left|\varepsilon_{J K L}\right| c_{K L}$ & 3 \\
\hline$\tilde{c}_{T, J}$ & $m\left(c_{T J}+c_{J T}\right)$ & 3 \\
\hline$\tilde{c}_{T T}$ & $m c_{T T}$ & 1 total: 44 \\
\hline
\end{tabular}


The keys to relating laboratory atomic clock data to the SME parameters are twofold:

- Understanding how the instantaneous atomic energy levels depend on the SME coefficients

- Understanding how the lab-frame coefficients change as the laboratory rotates and revolves.

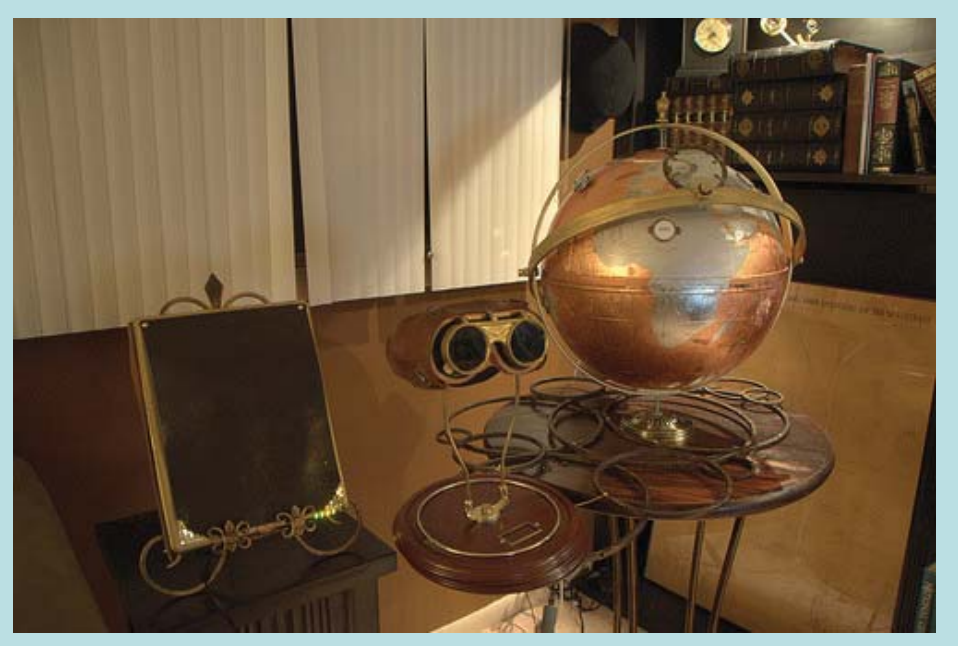


Some coefficients, such as $\tilde{b}_{j}$, are relatively easy to constrain with atomic clocks, as they affect the energies of spin substates directly.

$$
\delta H=-\tilde{b}_{j} \sigma^{j}
$$

Other are much harder to measure. These include the $c_{0 j}-\frac{1}{2} \tilde{k}_{0 j}$, because they are odd under $\mathrm{P}$ but spin-independent. They primarily affect the one-way velocities of particles. 
Neutron and proton coefficients are also easier to bound than electron ones, because most clock transitions use nuclear spins.

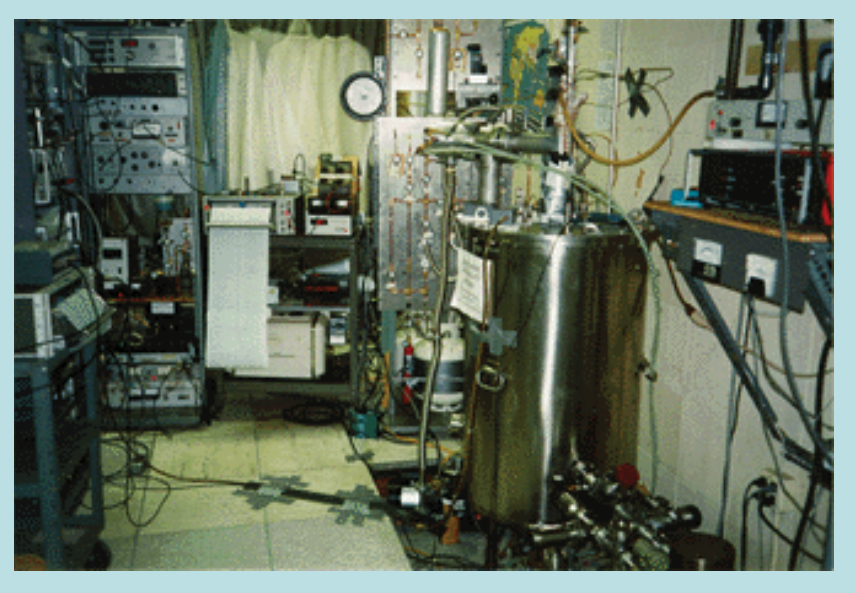

However, calculating the nuclear energy shifts requires matrix elements which can usually only be determined in the context of a simplified model. 
A typical simplification is the Schmidt model. For an odd-even nucleus (like ${ }^{3} \mathrm{He}$ and ${ }^{129} \mathrm{Xe}$ ), the model assigns all nuclear angular momentum to the single unpaired nucleon.

This model gives reasonable (but by no means excellent) agreement with magnetic moments.
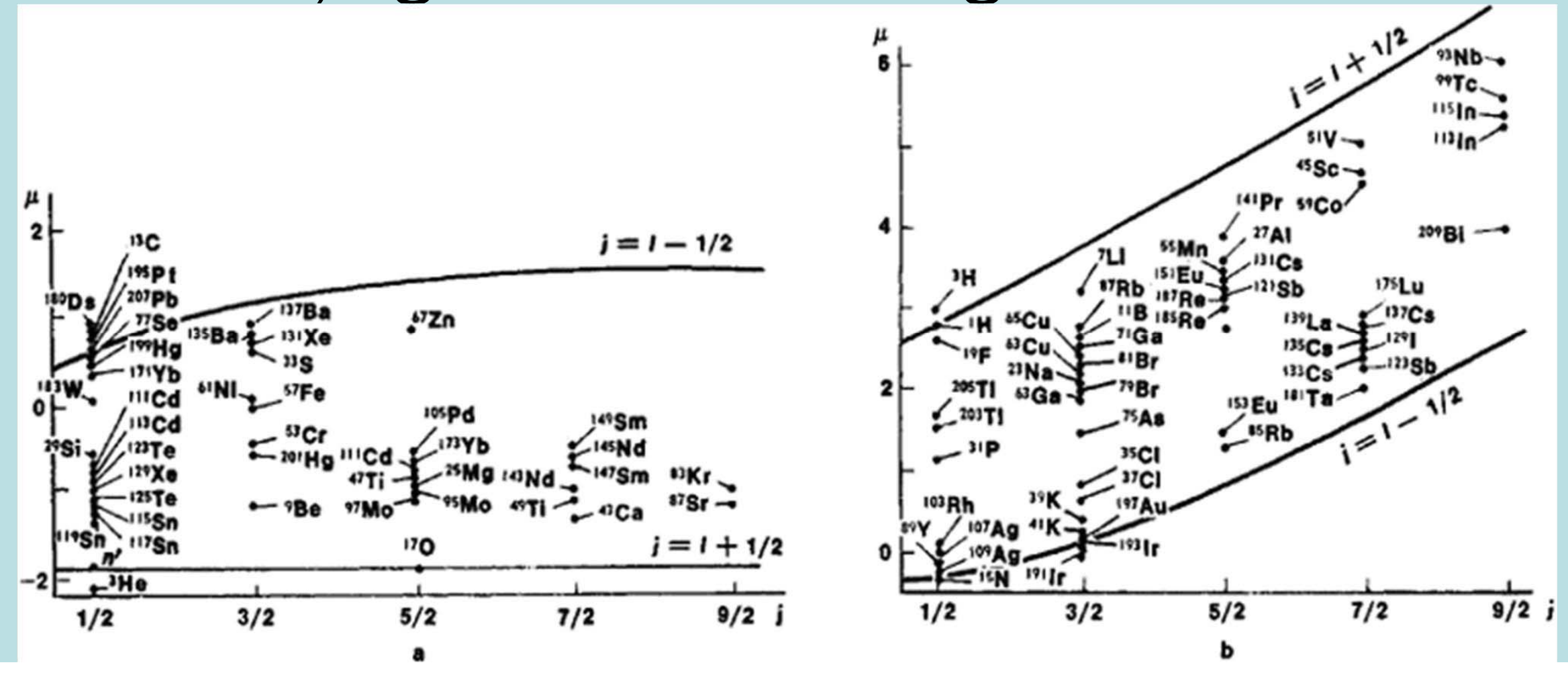
Evaluation of atomic and nuclear matrix elements for the unusual operators that appear in the Hamiltonian can be tricky, even with the Schmidt model simplification.

$$
\begin{aligned}
\delta \omega= & s_{1}^{p}\left[\beta_{p}\left(l_{j}\right) \tilde{b}_{3}^{p}+\delta_{p}\left(l_{j}\right) \tilde{d}_{3}^{p}+\kappa_{p}\left(l_{j}\right) \tilde{g}_{d}^{p}\right] \\
& +s_{2}^{p}\left[\gamma_{p}\left(l_{j}\right) \tilde{c}_{q}^{p}+\lambda_{p}\left(l_{j}\right) \tilde{g}_{q}^{p}\right] \\
& +s_{1}^{e}\left[\beta_{e}\left(0_{1 / 2}\right) \tilde{b}_{3}^{e}+\delta_{e}\left(0_{1 / 2}\right) \tilde{d}_{3}^{e}+\kappa_{e}\left(0_{1 / 2}\right) \tilde{g}_{d}^{e}\right]
\end{aligned}
$$

This depends on the lab frame coefficients and the species-specific matrix elements. 


\section{The matrix elements have been calculated for some classic clock transitions.}

\begin{tabular}{|c|c|c|c|c|c|c|c|c|c|c|c|}
\hline transition & \begin{tabular}{|c}
${ }^{133} \mathrm{Cs}$ \\
$(4,0)$ \\
$\rightarrow(3,0)$ \\
\end{tabular} & \begin{tabular}{|c}
${ }^{133} \mathrm{Cs}$ \\
$(4,1)$ \\
$\rightarrow(3,1)$ \\
\end{tabular} & $\begin{array}{c}{ }^{133} \mathrm{Cs} \\
(4,-1) \\
\rightarrow(3,-1) \\
\end{array}$ & \begin{tabular}{|c}
${ }^{133} \mathrm{Cs}$ \\
$(4,0)$ \\
$\rightarrow(3,1)$ \\
\end{tabular} & $\begin{array}{c}{ }^{133} \mathrm{Cs} \\
(4,0) \\
\rightarrow(3,-1) \\
\end{array}$ & \begin{tabular}{|c}
${ }^{87} \mathrm{Rb}$ \\
$(2,1)$ \\
$\rightarrow(1,1)$ \\
\end{tabular} & $\begin{array}{c}{ }^{87} \mathrm{Rb} \\
(2,-1) \\
\rightarrow(1,-1)\end{array}$ & \begin{tabular}{|c|c}
${ }^{87} \mathrm{Rb}$ \\
$(2,1)$ \\
$\rightarrow(1,0)$ \\
\end{tabular} & \begin{tabular}{|c|}
${ }^{87} \mathrm{Rb}$ \\
$(2,0)$ \\
$\rightarrow(1,1)$ \\
\end{tabular} & $\begin{array}{c}{ }^{87} \mathrm{Rb} \\
(2,0) \\
\rightarrow(1,-1) \\
\end{array}$ & $\begin{array}{c}{ }^{1} \mathrm{H} \\
(1,1) \\
\rightarrow(1,0) \\
\end{array}$ \\
\hline$I$ & $7 / 2$ & $7 / 2$ & $7 / 2$ & $7 / 2$ & $7 / 2$ & $3 / 2$ & $3 / 2$ & $3 / 2$ & $3 / 2$ & $3 / 2$ & $1 / 2$ \\
\hline$Z$ & 55 & 55 & 55 & 55 & 55 & 37 & 37 & 37 & 37 & 37 & 1 \\
\hline$N$ & 78 & 78 & 78 & 78 & 78 & 50 & 50 & 50 & 50 & 50 & 0 \\
\hline $\begin{array}{c}\text { Schmidt } \\
\text { nucleon }\end{array}$ & $g_{7 / 2}$ & $g_{7 / 2}$ & $g_{7 / 2}$ & $g_{7 / 2}$ & $g_{7 / 2}$ & $p_{3 / 2}$ & $p_{3 / 2}$ & $p_{3 / 2}$ & $p_{3 / 2}$ & $p_{3 / 2}$ & $s_{1 / 2}$ \\
\hline$e^{-}$state & $s_{1 / 2}$ & $s_{1 / 2}$ & $s_{1 / 2}$ & $s_{1 / 2}$ & $s_{1 / 2}$ & $s_{1 / 2}$ & $s_{1 / 2}$ & $s_{1 / 2}$ & $s_{1 / 2}$ & $s_{1 / 2}$ & $s_{1 / 2}$ \\
\hline $\begin{array}{c}\beta_{p} \\
\gamma_{p} \\
\delta_{p} \\
\kappa_{p} \\
\lambda_{p}\end{array}$ & $\begin{array}{c}{\left[\frac{7}{9}\right]} \\
{\left[-\frac{1}{9} K_{p}\right]} \\
{\left[-\frac{7}{33} K_{p}\right]} \\
{\left[\frac{28}{99} K_{p}\right]} \\
{[0]} \\
\end{array}$ & $\begin{array}{c}{\left[\frac{7}{9}\right]} \\
{\left[-\frac{1}{9} K_{p}\right]} \\
{\left[-\frac{7}{33} K_{p}\right]} \\
{\left[\frac{28}{99} K_{p}\right]} \\
{[0]} \\
\end{array}$ & $\begin{array}{c}{\left[\frac{7}{9}\right]} \\
{\left[-\frac{1}{9} K_{p}\right]} \\
{\left[-\frac{7}{33} K_{p}\right]} \\
{\left[\frac{28}{99} K_{p}\right]} \\
{[0]} \\
\end{array}$ & $\begin{array}{c}{\left[\frac{7}{9}\right]} \\
{\left[-\frac{1}{9} K_{p}\right]} \\
{\left[-\frac{7}{33} K_{p}\right]} \\
{\left[\frac{28}{99} K_{p}\right]} \\
{[0]} \\
\end{array}$ & $\begin{array}{c}{\left[\frac{7}{9}\right]} \\
{\left[-\frac{1}{9} K_{p}\right]} \\
{\left[-\frac{7}{33} K_{p}\right]} \\
{\left[\frac{28}{99} K_{p}\right]} \\
{[0]} \\
\end{array}$ & $\begin{array}{c}{[-1]} \\
{\left[-\frac{1}{15} K_{p}\right]} \\
{\left[\frac{1}{5} K_{p}\right]} \\
{\left[-\frac{2}{5} K_{p}\right]} \\
{[0]} \\
\end{array}$ & $\begin{array}{c}{[-1]} \\
{\left[-\frac{1}{15} K_{p}\right]} \\
{\left[\frac{1}{5} K_{p}\right]} \\
{\left[-\frac{2}{5} K_{p}\right]} \\
{[0]} \\
{[0]}\end{array}$ & $\begin{array}{c}{[-1]} \\
{\left[-\frac{1}{15} K_{p}\right]} \\
{\left[\frac{1}{5} K_{p}\right]} \\
{\left[-\frac{2}{5} K_{p}\right]} \\
{[0]} \\
\end{array}$ & $\begin{array}{c}{[-1]} \\
{\left[-\frac{1}{15} K_{p}\right]} \\
{\left[\frac{1}{5} K_{p}\right]} \\
{\left[-\frac{2}{5} K_{p}\right]} \\
{[0]} \\
\end{array}$ & $\begin{array}{c}{[-1]} \\
{\left[-\frac{1}{15} K_{p}\right]} \\
{\left[\frac{1}{5} K_{p}\right]} \\
{\left[-\frac{2}{5} K_{p}\right]} \\
{[0]}\end{array}$ & $\begin{array}{c}-1 \\
0 \\
\frac{1}{3} K_{p} \\
-\frac{1}{3} K_{p} \\
0 \\
\end{array}$ \\
\hline$\beta_{e}$ & -1 & -1 & -1 & -1 & -1 & -1 & -1 & -1 & -1 & -1 & -1 \\
\hline$\gamma_{e}$ & 0 & 0 & 0 & 0 & 0 & 0 & 0 & 0 & 0 & 0 & 0 \\
\hline$\delta_{e}$ & $\frac{1}{3} K_{e}$ & $\frac{1}{3} K_{e}$ & $\frac{1}{3} K_{e}$ & $\frac{1}{3} K_{e}$ & $\frac{1}{3} K_{e}$ & $\frac{1}{3} K_{e}$ & $\frac{1}{3} K_{e}$ & $\frac{1}{3} K_{e}$ & $\frac{1}{3} K_{e}$ & $\frac{1}{3} K_{e}$ & $\frac{1}{3} K_{e}$ \\
\hline $\begin{array}{l}\kappa_{e} \\
\lambda_{e}\end{array}$ & $\begin{array}{c}-\frac{1}{3} K_{e} \\
0\end{array}$ & $\begin{array}{c}-\frac{1}{3} K_{e} \\
0\end{array}$ & $\begin{array}{c}-\frac{1}{3} K_{e} \\
0\end{array}$ & $\begin{array}{c}-\frac{1}{3} K_{e} \\
0\end{array}$ & $\begin{array}{c}-\frac{1}{3} K_{e} \\
0\end{array}$ & $\begin{array}{c}-\frac{1}{3} K_{e} \\
0\end{array}$ & $\begin{array}{c}-\frac{1}{3} K_{e} \\
0\end{array}$ & $\begin{array}{c}-\frac{1}{3} K_{e} \\
0\end{array}$ & $\begin{array}{c}-\frac{1}{3} K_{e} \\
0\end{array}$ & $\begin{array}{c}-\frac{1}{3} K_{e} \\
0\end{array}$ & $\begin{array}{c}-\frac{1}{3} K_{e} \\
0\end{array}$ \\
\hline$s_{1}^{p}$ & 0 & $-1 / 14$ & $1 / 14$ & $-9 / 28$ & $9 / 28$ & $-1 / 3$ & $1 / 3$ & $1 / 2$ & $-5 / 6$ & $5 / 6$ & 1 \\
\hline$s_{2}^{p}$ & 0 & $-1 / 14$ & $-1 / 14$ & $-5 / 28$ & $-5 / 28$ & -1 & -1 & $1 / 2$ & $-3 / 2$ & $-3 / 2$ & - \\
\hline$s_{1}^{e}$ & 0 & $1 / 2$ & $-1 / 2$ & $1 / 4$ & $-1 / 4$ & 1 & -1 & $1 / 2$ & $1 / 2$ & $-1 / 2$ & 1 \\
\hline
\end{tabular}


The energy shifts will change with the orientation of the earthbound laboratory.

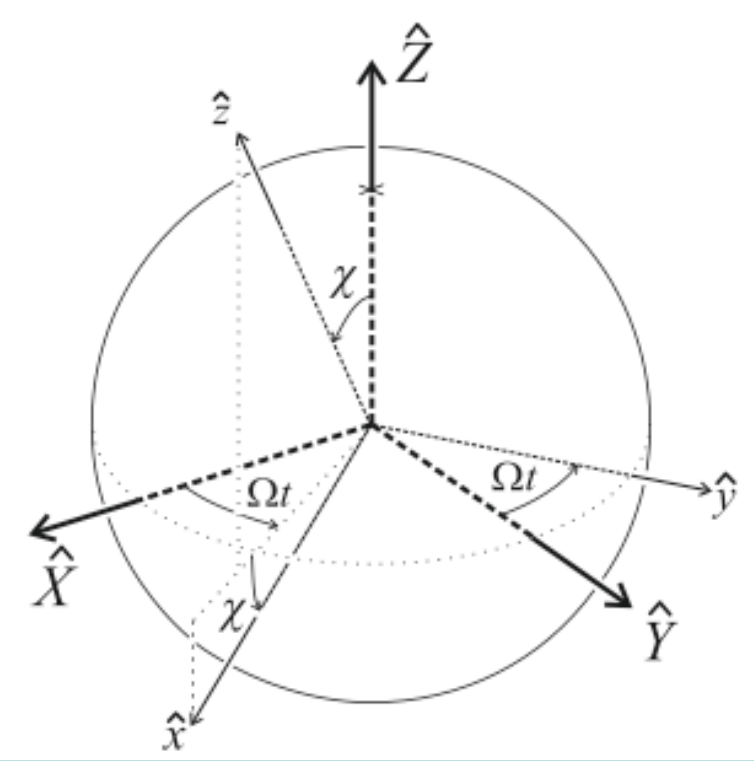

$$
\left(\begin{array}{c}
\hat{x} \\
\hat{y} \\
\hat{z}
\end{array}\right)=\left(\begin{array}{ccc}
\cos \chi \cos \Omega t & \cos \chi \sin \Omega t & -\sin \chi \\
-\sin \Omega t & \cos \Omega t & 0 \\
\sin \chi \cos \Omega t & \sin \chi \sin \Omega t & \cos \chi
\end{array}\right)\left(\begin{array}{c}
\hat{X} \\
\hat{Y} \\
\hat{Z}
\end{array}\right)
$$

Bounds are usually quoted in celestial, equatorial, suncentered coordinates $(X, Y, Z, T)$ that are approximately inertial.

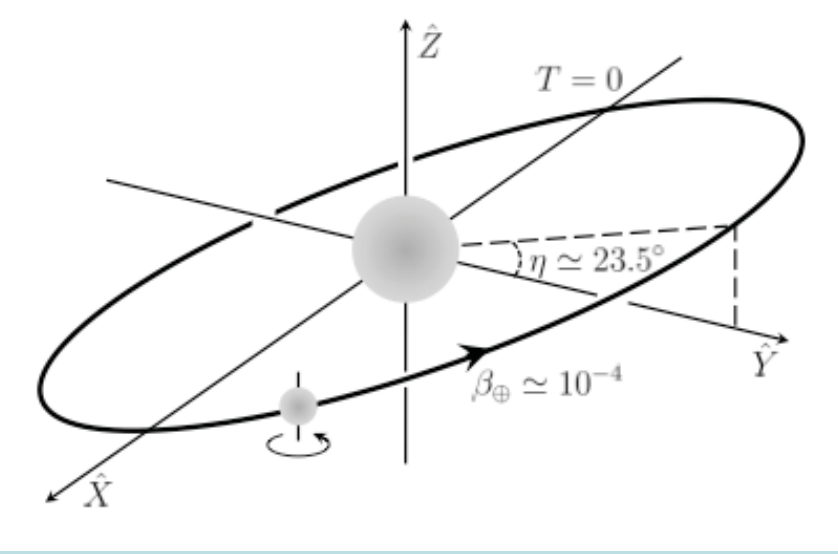


The full transformations from the sun-centered frame to a laboratory frame (including the effects of boosts) get pretty complicated.

$$
\begin{aligned}
& b_{3}^{w}=b_{T}^{w}\left\{\beta_{s}-\right. \beta_{\oplus}\left[\operatorname { s i n } \Omega _ { \oplus } T \left(\cos \alpha \sin \omega_{s} \Delta T\right.\right. \\
&\left.+\cos \zeta \sin \alpha \cos \omega_{s} \Delta T\right)-\cos \eta \cos \Omega_{\oplus} T \\
& \times\left(\sin \alpha \sin \omega_{s} \Delta T-\cos \zeta \cos \alpha \cos \omega_{s} \Delta T\right) \\
&\left.\left.+\sin \eta \cos \Omega_{\oplus} T \sin \zeta \cos \omega_{s} \Delta T\right]\right\} \\
&-b_{X}^{w}\left(\cos \alpha \sin \omega_{s} \Delta T+\cos \zeta \sin \alpha \cos \omega_{s} \Delta T\right) \\
&-b_{Y}^{w}\left(\sin \alpha \sin \omega_{s} \Delta T-\cos \zeta \cos \alpha \cos \omega_{s} \Delta T\right) \\
&+b_{Z}^{w} \sin \zeta \cos \omega_{s} \Delta T
\end{aligned}
$$

This expression is for a hypothetical future clock on a satellite, so it depends on the satellite velocity, the satellite's orbital incliation, and the revolution of the Earth. 
The energy shifts oscillate at the sidereal rotation period of the Earth.

If measurements

are made over many

months, noise

associated with

day/night

differences in the

environment

average out.

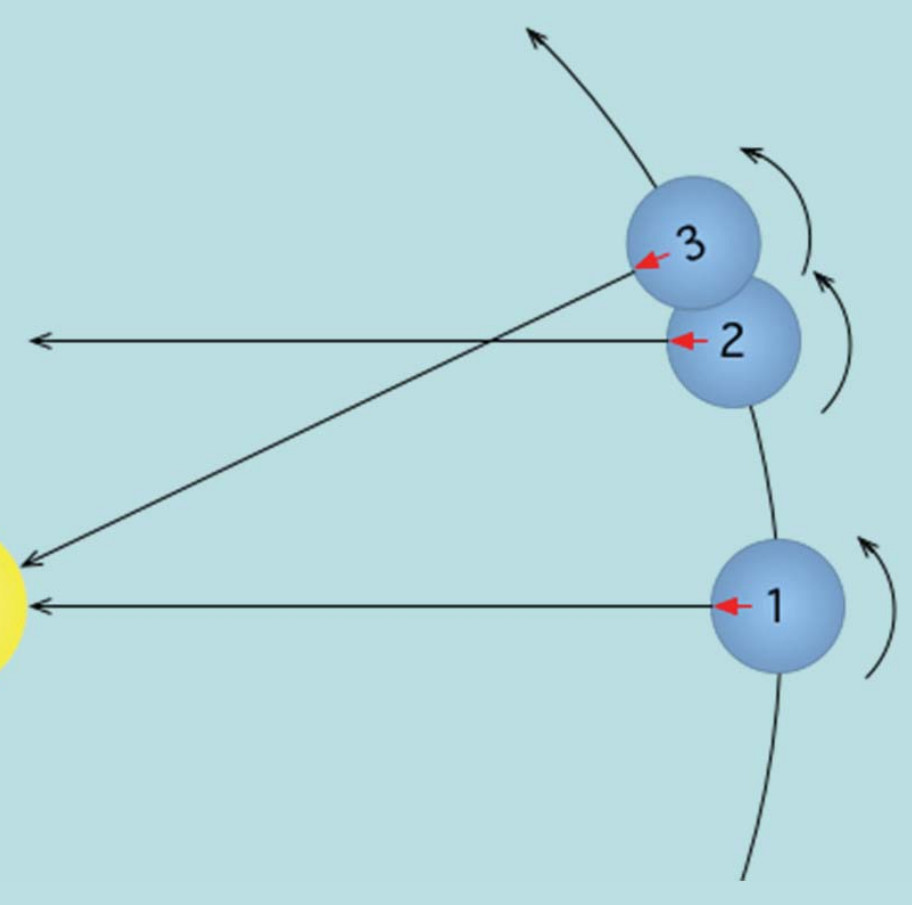


Over the course of a year, the boost of the Earth changes, opening up sensitivity to other coefficients (with $v_{\oplus} \approx 10^{-4}$ suppression).

$$
\tilde{b}_{j}^{l a b} \approx \tilde{b}_{J}+\left(v_{\oplus}\right)_{j} \tilde{b}_{T}
$$

This effect can be used in two ways:

- Search for yearly variations in a transition frequency

- Search for yearly variations in the amplitude of sidereal frequency modulations 
The cleanest measurements of $\tilde{b}_{J}$ typically come from two-species frequency comparisons.

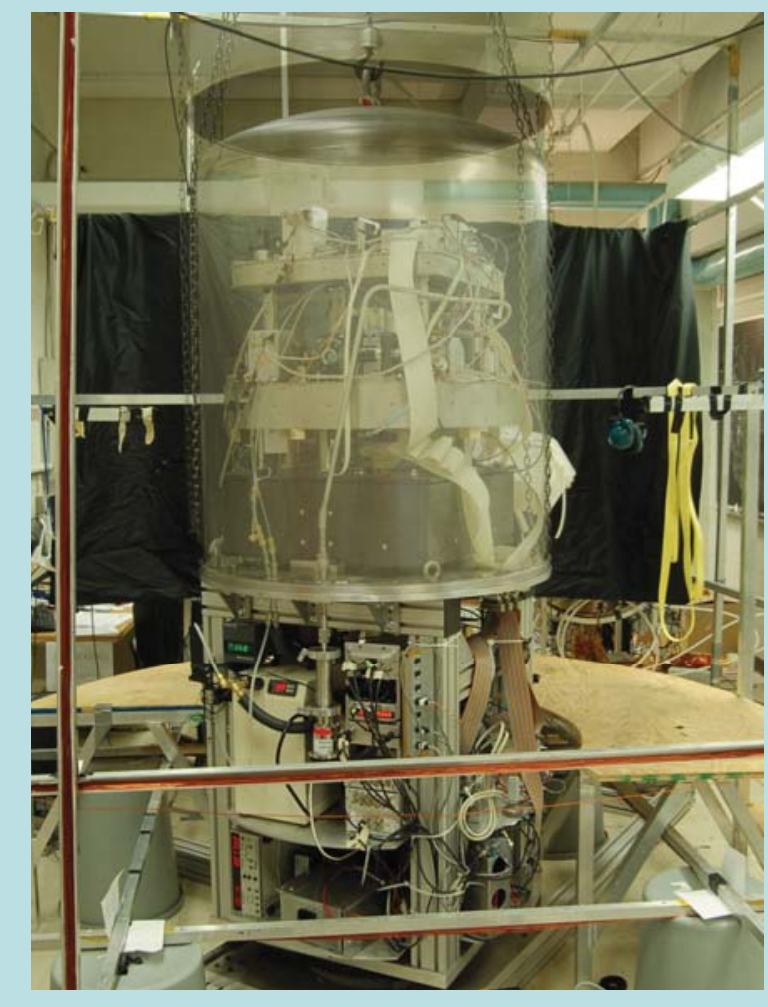

$\mathrm{K}-{ }^{3} \mathrm{He}$ clock at Princeton
Typically, one species is polarized directly, while the other is polarized by spin transfer.

Then transition frequencies for both species are read out and compared. 
The net bounds are typically at the $10^{-31} \mathrm{GeV}$ level for the electron, proton, and neutron $\tilde{b}_{J}$ coefficients.

Other types of coefficients are less comprehensively constrained. A lot more work can be done in this area.

We will see that it is also advantageous to have multiple bounds on the $\tilde{b}_{J}$, using different systems. 


\section{Ideas for Future Improvements}

Since atomic clocks offer so much precision, we would really like to get the most possible results for clock experiments.

There are many possible avenues for improvement, both using existing clocks and new clock setups. 
Some of the possible improvements give us access to different SME coefficients by considering more motions for the laboratory.

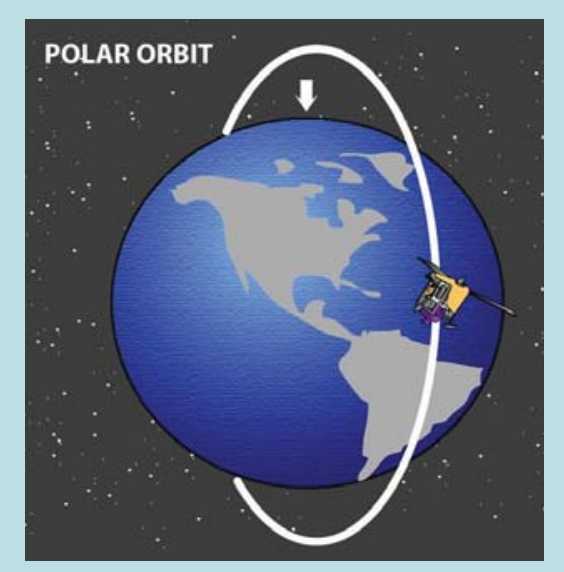

If atomic clocks are placed on Earth-orbiting satellites, that will lead to immediate improvements in sensitivity. 
Actually, sensitivity to the additional coefficients already exists in Earthbound labs (although it is strongly suppressed).

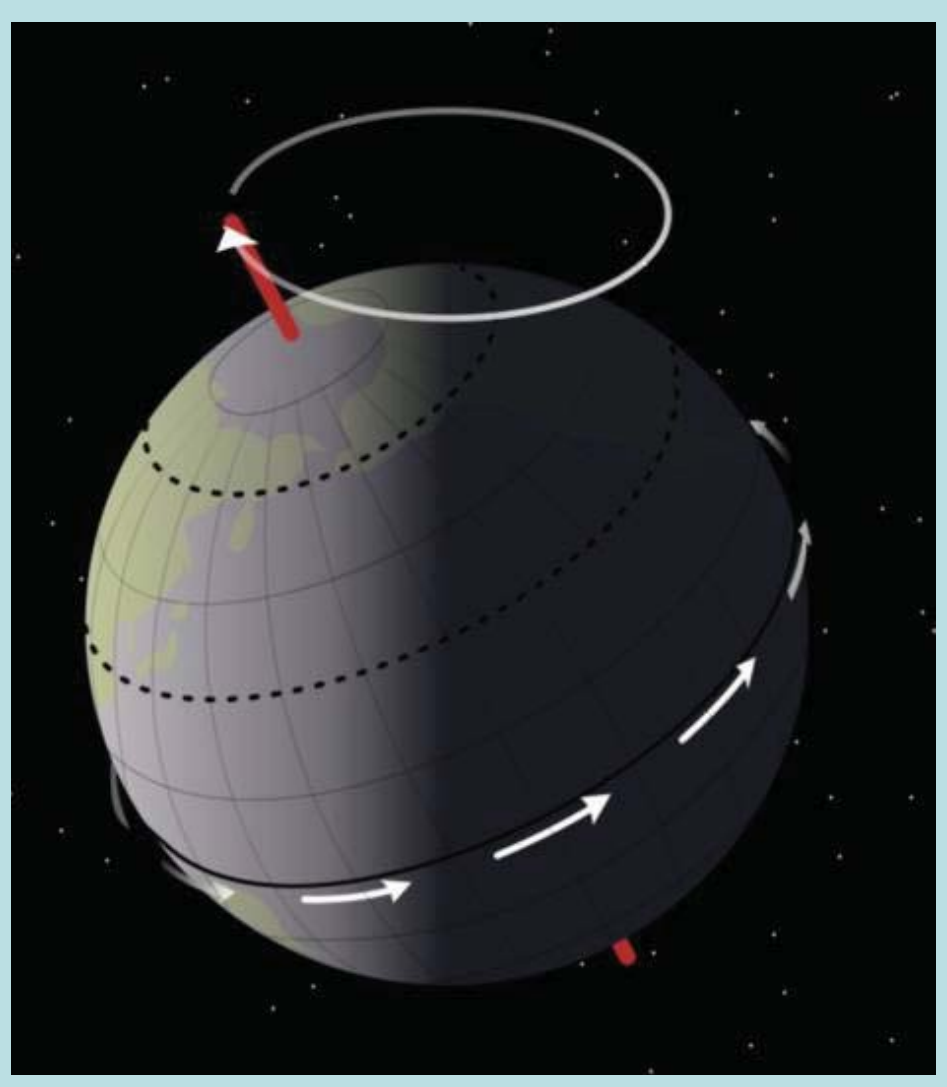

With the precession of the equinoxes, the Earth's rotation axis moves by $10^{-4}$ per year.

[Hipparchus, On the Displacement of the Solstice and Equinox Points (ca. 135 BCE)] 
Another idea is to make use of the weak relativistic effects in atoms and nuclei to get sensitivity to additional linear combinations of coefficients.

If we measure the Lorentz-violating spin coupling for a particle with binding energy $e$ and kinetic energy $e_{K}$, we actually measure

$\stackrel{\circ}{b}_{J}(e)=b_{J}-\frac{1}{2}\left(1+e_{K} / 3 m\right) \epsilon_{J K L} H_{K L}-\left(m-e-2 e_{K} / 3\right) d_{J T}+\frac{1}{2}\left(m-e-e_{K}\right) \epsilon_{J K L} g_{K L T}$. 
Another way to add sensitivity is to go beyond the Schmidt model.

Unless protons or neutrons form a closed shell, they will make some net contribution to the total nuclear spin. For small $A$ nuclei, these can be calculated reasonably well.

These are two reasons to repeat clock comparisons in similar but distinct atomic systems.

For example: ${ }^{3} \mathrm{He} /{ }^{129} \mathrm{Xe}$ versus ${ }^{3} \mathrm{He} / \mathrm{K}$ versus $\mathrm{H}$ 


\section{Other Exotic Possibilities}

Many other exotic phenomena can be tested with atomic clocks, and many of these are related to Lorentz and CPT violations.

For example, the 1S-2S hydrogen transition was already used to constrain $\dot{\alpha}$.

A nonzero $\dot{\alpha}$ can also give rise to boost invariance violation (since the derivative selects a preferred timelike direction). 
Tests have also been made of the dependence of fundamental constants on the gravitational field or the Earth-Sun distance.

\section{[Blatt, et al., PRL 100, 140801 (2008)]}

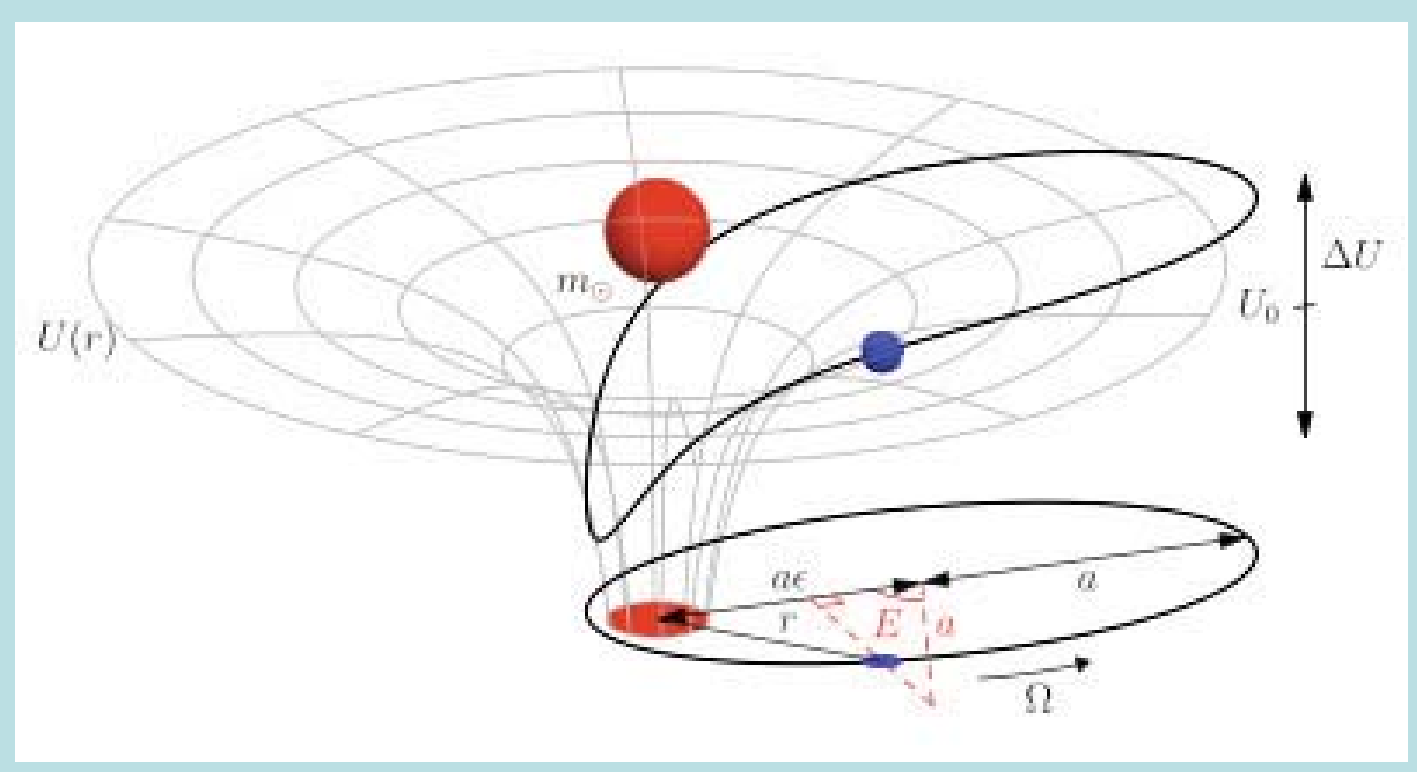

Many other possible experiments are possible. 


\section{Conclusion}

Atomic clocks offer one of the best ways to test Lorentz symmetry.

Nonrelativistic tests of anisotropy are sensitive to specific linear combinations of SME coefficients.

With different systems and including small relativistic effects, significant improvements over existing bounds are possible. 
Thanks to V. A. Kostelecký and E. Pfister-Altschul.
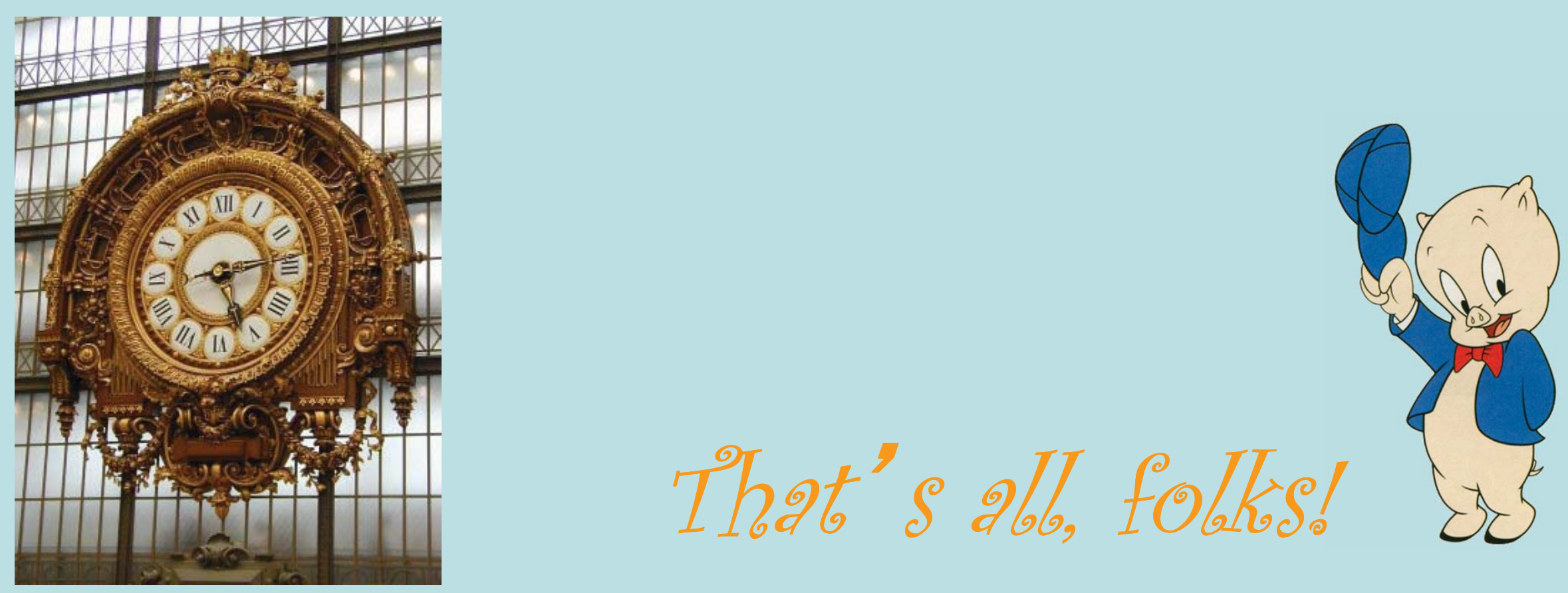